\title{
Zariski dense surface subgroups in $\operatorname{SL}(3, \mathrm{Z})$
}

\author{
DARREN D LONG \\ ALAN W REID \\ MORWEN THISTLETHWAITE
}

\begin{abstract}
We exhibit an infinite family of Zariski dense surface groups of fixed genus inside $\mathrm{SL}(3, \mathbf{Z})$.
\end{abstract}

22E40, 20H10

\section{Introduction}

This paper proves the existence of an infinite family of Zariski dense surface subgroups of fixed genus inside $\operatorname{SL}(3, \mathbf{Z})$. To put this into context, we recall that it follows from Tits [9] that free groups are plentiful in $\operatorname{SL}(3, \mathbf{Z})$ and, moreover, it is not difficult to see that (one can use Theorem 2.2 below, for example) it is easily arranged that these free groups are Zariski dense. Less trivially, classical arithmetic considerations (see for example Long and Reid [6, Section 6.1]) can be used to construct surface subgroups of $\operatorname{SL}(3, \mathbf{Z})$ of every genus $\geq 2$. However these are constructed using the theory of quadratic forms, so that their Zariski closures in $\operatorname{SL}(3, \mathbf{R})$ are $\mathrm{SO}(f, \mathbf{R})$ for some appropriate ternary quadratic form $f$; in particular these surface groups are not Zariski dense in $\operatorname{SL}(3, \mathbf{R})$. With this as background we state the main result of this note:

Theorem 1.1 The family of representations of the triangle group

$$
\begin{aligned}
\Delta(3,3,4) & =\left\langle a, b \mid a^{3}=b^{3}=(a . b)^{4}=1\right\rangle \\
a & \mapsto\left(\begin{array}{lll}
0 & 0 & 1 \\
1 & 0 & 0 \\
0 & 1 & 0
\end{array}\right) \\
b & \mapsto\left(\begin{array}{ccc}
1 & 2-t+t^{2} & 3+t^{2} \\
0 & -2+2 t-t^{2} & -1+t-t^{2} \\
0 & 3-3 t+t^{2} & (-1+t)^{2}
\end{array}\right)
\end{aligned}
$$

are discrete and faithful for every $t \in \mathbf{R}$.

Moreover, for all integral values of $t$ the image groups are nonconjugate subgroups of $\operatorname{SL}(3, \mathbf{Z})$ which are Zariski dense in $\operatorname{SL}(3, \mathbf{R})$. 
Since for any $t \in \mathbf{R}$, these subgroups are all isomorphic to $\Delta(3,3,4)$, by taking any $\mathbf{Z}$ specialization of $t$ and passing to a subgroup of finite index, we obtain a family of Zariski dense surface groups (for every fixed genus $\geq 2$ ) inside $\operatorname{SL}(3, \mathbf{Z})$.

To the authors' knowledge, this is the first family of nonconjugate, infinite index, Zariski dense freely indecomposable subgroups of $\operatorname{SL}(3, \mathbf{Z})$ that has been constructed. In fact, as far as we know there was only one such subgroup known previously, due to Kac and Vinberg [10] which was produced by a completely different method. This appears in our family as the case $t=1$ (after a suitable conjugation). It is our understanding that the method of [10] is restricted to producing at most a finite number of such representations.

The representations given by Theorem 1.1 lie on the so-called Hitchin component (see Choi and Goldman [3] and Section 2) of representations of $\Delta(3,3,4)$ into $\operatorname{SL}(3, \mathbf{R})$, and it follows that any element of infinite order necessarily has three distinct real eigenvalues (see [3] and Goldman [5]). For convenience, we will refer to these as purely semisimple groups. Recall from Serre [8] that the group $\Delta(3,3,4)$ has Property FA. Hence we have the following corollary.

Corollary 1.2 There exists an infinite family of nonconjugate Zariski dense, infinite index, freely indecomposable, purely semisimple subgroups of $\operatorname{SL}(3, \mathbf{Z})$ isomorphic to a fixed group $G$ which is word hyperbolic and has Property FA.

In general, the existence of a family of subgroups with the properties as given by Corollary 1.2 seem rare - indeed, there are many results having the flavour that the number of conjugacy classes of images of a fixed group $G$ into target groups $\Gamma$ that are constrained to have various geometric properties is finite. For example, the corollary is in striking contrast to Bowditch [2], which shows that if $\Gamma$ is a mapping class group, then this has only finitely many conjugacy classes of purely pseudo-Anosov surface subgroups.

Our results should also be compared with Wang [11], which produces families of faithful discrete representations of surface groups into $\operatorname{SL}(n, \mathbf{R})$ for $n \geq 3$. However, as is pointed out in [11], the representations constructed in [11] do not lie in the Hitchin component. Furthermore, his methods do not produce representations into $\operatorname{SL}(3, \mathbf{Z})$. And while his methods do produce representations into $\operatorname{SL}(n, \mathbf{Z})$ for $n \geq 5$, it is not easily checked whether these give purely semisimple groups.

Roughly, the method of proof of Theorem 1.1 is to exploit convex real projective structures. For, it is known from [3], that if $\Delta(p, q, r)$ is a hyperbolic triangle group with $p, q, r$ all greater than 2 , then the space $\operatorname{Hom}(\Delta(p, q, r), \operatorname{PGL}(3, \mathbf{R})) / \operatorname{PGL}(3, \mathbf{R})$ 
contains a certain two dimensional component (the so-called Hitchin component $\left.X(p, q, r)^{\mathrm{Hit}}\right)$, which is characterised by the property that the corresponding representations are precisely those that determine convex real projective structures on the quotient 2-orbifold. In particular, our use of this is the powerful fact that every such representation is discrete and faithful. This is then exploited via the method of [4], which computes the representation variety and identifies this component explicitly. Taken together with some elementary Diophantine analysis, this yields the curve of representations defined above.

For character reasons, the values of $p, q, r$ for which a hyperbolic triangle group could have a faithful representation into $\operatorname{SL}(3, \mathbf{Z})$ must be drawn from $\{2,3,4,6, \infty\}$ and our method works in these cases also. For example, in Section 3, we include a discrete faithful family of Zariski dense representations of the triangle group $\Delta(3,4,4)$.

Acknowledgements The authors are grateful to the referee for a careful reading of the original version of this paper, and for offering many constructive comments which have improved the exposition.

We are indebted to W Goldman for introducing us to the example of [10].

\section{The construction}

Using the methods of [4], one can prove that the two dimensional real family of representations of the triangle group $\Delta(3,3,4)$ which is predicted by [3] is given by

$$
\begin{aligned}
& a \mapsto\left(\begin{array}{ccc}
1 & 1 & u\left(-u^{3} v+4 u v^{2}-2 v^{3}+u^{2}(-6 v+\sqrt{D})\right) / \tau \\
0 & -v / u & 1 \\
0 & \left(-u^{2}+u v-v^{2}\right) / u^{2} & -1+v / u
\end{array}\right) \\
& b \mapsto\left(\begin{array}{ccc}
-1+v / u & 0 & u\left(2 u^{3}+2 v^{3}-u^{2} v(2+v)\right. \\
+u v(-2 v+\sqrt{D})) / \tau \\
\left(-2 v^{2}+u^{2}(2+v)\right. & & -1 \\
+u(4 v+\sqrt{D})) /\left(2 u^{2}\right) & 1 & \\
\left(-2 u^{3}-2 v^{3}+u^{2} v(2+v)\right. & 0 & -v / u \\
+u v(2 v+\sqrt{D})) /\left(2 u^{3}\right) & &
\end{array}\right)
\end{aligned}
$$

where

$$
\tau=2\left(u^{2}+v^{2}\right)\left(u^{2}-u v+v^{2}\right)
$$

and

$$
D=-4 u^{2}(5+u)+4 u(8+u) v+(-20+u(4+u)) v^{2}-4 v^{3} .
$$

The hyperbolic representation occurs in the above matrices at $u=2 \sqrt{2}, v=2 \sqrt{2}$. 
It is technical to describe the method of [4] in any detail. Very roughly, one computes the hyperbolic representation of $\Delta(3,3,4)$ into $\operatorname{SO}(2,1, \mathbf{R})$ and then uses Newton's method to compute to high accuracy a large number of nearby representations into $\mathrm{SL}(3, \mathbf{R})$. One then couples the algorithm of Lenstra-Lenstra-Lovacs with a polynomial interpolation method to compute the polynomials defining the field extensions for the matrix entries over the field $\mathbf{Q}(u, v)$, where $u$ and $v$ are carefully chosen matrix traces. As remarked in the introduction, it follows from [3] that there is a certain two (real) dimensional component $X(3,3,4)^{\text {Hit }}$ where all the representations define convex real projective structures. In particular, all the representations on this component are discrete and faithful.

This picture is reflected in our representation family as follows. There is a discriminant

$$
D=-4 u^{2}(5+u)+4 u(8+u) v+(-20+u(4+u)) v^{2}-4 v^{3}
$$

that needs to be positive in order that the matrix entries be real. When one plots the locus $D=0$ in the $u v$-plane it appears as in Figure 1 below.

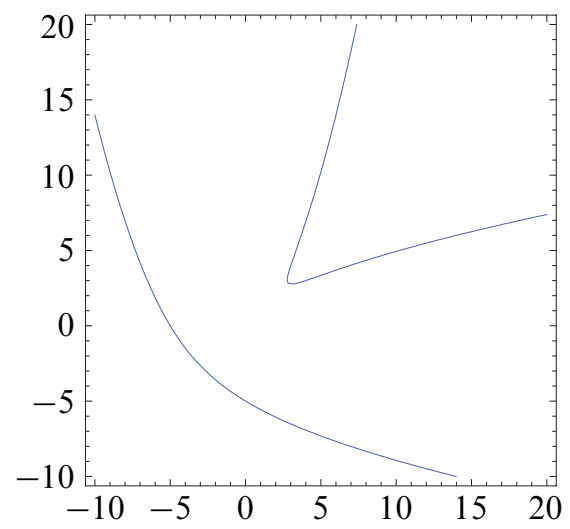

Figure 1

Each value in the regions where $D>0$ corresponds to two representations, depending on which sign one takes for $\sqrt{D}$. In this way one sees that each of the two components is a topological disc. (See [3].)

The triangle group $\Delta(3,3,4)$ admits one interesting representation into $\operatorname{SO}(2,1 ; \mathbf{R})$ up to conjugacy; this representation is discrete and faithful. Of course, this can then be embedded in $\operatorname{SL}(3, \mathbf{R})$ in more than one way. In our family, the point $(2 \sqrt{2}, 2 \sqrt{2})$ can be shown to define the hyperbolic embedding, as this preserves a form of signature $(2,1)$. This representation appears (as it must by uniqueness) on the locus $D=0$. It follows that, given the structure we have set forth above, that all the representations in 
the quadrant $u>0, v>0$ and which lie in the region $D \geq 0$ correspond to discrete faithful representations of $\Delta(3,3,4)$ (since they correspond to points in $X(3,3,4)^{\mathrm{Hit}}$ ). This completes the first part of the proof of Theorem 1.1.

We now focus on which of these representations can be conjugated to be integral. To this end one computes that the characteristic polynomial of the element $a \cdot b^{-1}$ is

$$
1-Q^{3}-Q(2+u)+Q^{2}(2+v)
$$

and for $[a, b]$ it is

$$
1-Q^{3}-Q(2+2 u+2 v+u v-\sqrt{D}) / 2+Q^{2}(2+2 u+2 v+u v-\sqrt{D}) / 2
$$

from which it follows that it is a necessary condition to restrict to those rational integers $u, v$ making $D$ a rational square.

A complete Diophantine analysis of this situation seems rather complicated as it involves a study of the integral points on a complex surface $D(u, v)=r^{2}$, however a very simple-minded analysis using an interpolation method gives that if one takes $u=4-3 k+2 k^{2}$ and $v=6-5 k+2 k^{2}$, then $D=(-3+2 k)^{2}(-1+2 k)^{2}\left(4-2 k+k^{2}\right)^{2}$. This substitution yields a representation of $\Delta(3,3,4)$ which computations reveal has integral character.

One then finds by direct computation that this specialization yields a representation which can be conjugated into $\operatorname{SL}(3, \mathbf{Z})$.

We note parenthetically that the fact this can be done for a rational representation of integral character is guaranteed by the following proposition. This appears to be fairly well known (and moreover it is of a nonconstructive nature) so we defer the proof to the end of this section.

Proposition 2.1 Let $\Gamma$ be a finitely generated nonsolvable subgroup of $\operatorname{SL}(3, \mathbf{Q})$ for which $\operatorname{tr}(\gamma) \in \mathbf{Z}$ for all $\gamma \in \Gamma$.

Then $\Gamma$ is $\operatorname{GL}(3, \mathbf{Q})$-conjugate into $\operatorname{SL}(3, \mathbf{Z})$.

One finds in this way the family of (necessarily) discrete faithful representations of $\Delta(3,3,4)$ into $\operatorname{SL}(3, \mathbf{Z})$ described in the introduction. As mentioned in Section 1 , the representation found by Kac and Vinberg is a conjugate of that obtained by setting $t=1$.

We now turn to the proof of the Zariski density claim of Theorem 1.1; we emphasise that this aspect is rather unsurprising, since once one has verified that the groups of Theorem 1.1 preserve no form, there are essentially no other Lie subgroups of $\operatorname{SL}(3, \mathbf{R})$ possible for the Zariski closure. However the result can be seen directly by appealing to Theorem 2.7 of [6]: 
Theorem 2.2 Let $G$ be a finitely generated nonsoluble subgroup of $\operatorname{SL}(3, \mathbf{Z})$. Suppose that there is an element $g \in G$ whose characteristic polynomial is $\mathbf{Z}$-irreducible and noncyclotomic.

Then $G$ is Zariski dense in $\operatorname{SL}(3, \mathbf{Z})$.

We will apply this in the following way. The characteristic polynomial of $[a, b]$ is

$$
p(Q)=1-Q^{3}+Q^{2}\left(17-8 t+4 t^{2}\right)+Q\left(-29+46 t-39 t^{2}+16 t^{3}-4 t^{4}\right) .
$$

It is clear that the polynomial $p(Q)$ cannot have a root \pm 1 for $t \gg 0$, so that by the Gauss Lemma, $p(Q)$ is irreducible for large $t$. Moreover, it cannot be cyclotomic when $17-8 t+4 t^{2}>3$, so that even this soft computation shows that the representations are Zariski dense for all but finitely many $t$. In fact an easy computer computation shows that $p(Q)$ satisfies the conditions of the theorem for all integral $t$. Thus the representations are Zariski dense for all integral $t$.

Notice the only elements of order three in $\Delta(3,3,4)$ are conjugate to the elements $a^{ \pm 1}$ and $b^{ \pm 1}$, so that $p(Q)$ is an invariant of conjugacy and automorphisms of the triangle group. It follows that our family is indeed a family of distinct representations. This completes the proof of Theorem 1.1.

For completeness, we sketch:

Proof of Proposition 2.1 Following Bass [1], consider the set

$$
\mathcal{O} \Gamma=\left\{\sum a_{i} \gamma_{i} \mid a_{i} \in \mathbf{Z}, \gamma_{i} \in \Gamma \text {, where the sum is finite }\right\} .
$$

As is shown in [1, Proposition 2.2 and Corollary 2.3], this is an order of a central simple algebra $B$ defined over $\mathbf{Q}$ (ie $\mathcal{O} \Gamma$ is a finitely generated $\mathbf{Z}$-module which contains a $\mathbf{Q}$-basis for $B$ and is also a ring with identity). Since the dimension of such an algebra is a square, it follows that $B$ either has dimension 4 or 9 . In the latter case, $B=M(3, \mathbf{Q})$, and so $\mathcal{O} \Gamma$ is an order of $M(3, \mathbf{Q})$. In the former case, it is to easy to see that we can always embed this order in an order of $M(3, \mathbf{Q})$ (a proof of this is sketched below). Thus, we will assume that $\mathcal{O} \Gamma$ is an order of $M(3, \mathbf{Q})$.

Now any order of $M(3, \mathbf{Q})$ is contained in a maximal order of $M(3, \mathbf{Q})$ (see $[7$, Corollary 10.4]), and so the proposition is will easily follow from:

Claim Every maximal order of $M(3, \mathbf{Q})$ is $\operatorname{GL}(3, \mathbf{Q})$-conjugate to $M(3, \mathbf{Z})$.

Proof of Claim Every maximal order $\mathcal{D}$ of $M(3, \mathbf{Q})$ can be described as follows (see [7, Theorem 21.6]). Let $V=\mathbf{Q}^{3}$ and identify $M(3, \mathbf{Q})$ with $\operatorname{End}(V)$. Then 
$\mathcal{D}=\{\sigma \in \operatorname{End}(V): \sigma(M) \subset M\}$ for some full $\mathbf{Z}$-lattice $M \subset V$ (ie a finitely generated $\mathbf{Z}$-submodule of $V$ which contains a $\mathbf{Q}$-basis for $V$ ).

Given this we argue as follows. Since $M$ is a full $\mathbf{Z}$-lattice, it is a torsion free $\mathbf{Z}$ module, and hence a free $\mathbf{Z}$-module. In particular, $\mathcal{D} \cong M(3, \mathbf{Z})$, and so $\mathcal{D} \otimes \mathbf{Q} \cong$ $M(3, \mathbf{Q})$. By the Skölem-Noether Theorem, such an isomorphism is given by a $\mathrm{GL}(3, \mathbf{Q})$-conjugation as required.

Finally we prove the embedding statement made above. Recall that if $M$ is a full $\mathbf{Z}$-lattice in $M(3, \mathbf{Q})$, the right order associated to $M$ is defined to be

$$
\mathcal{O}_{r}(M)=\{x \in M(3, \mathbf{Q}): M . x \subset M\} .
$$

This is an order of $M(3, \mathbf{Q})$ (see [7, page 109]).

Lemma 2.3 In the notation above, if $\mathcal{O} \Gamma$ is an order in $B$ a quaternion subalgebra of $M(3, \mathbf{Q})$, then there is an order $\mathcal{D} \subset M(3, \mathbf{Q})$ such that $\mathcal{O} \Gamma \subset \mathcal{D}$.

Proof Let $M$ be any full $\mathbf{Z}$ lattice in $M(3, \mathbf{Q})$. Since $\mathcal{O} \Gamma$ is an order of $B$, it follows that $M .(\mathcal{O} \Gamma)$ is also a full $\mathbf{Z}$-lattice of $M(3, \mathbf{Q})$. Let $\mathcal{D}$ denote the right order associated to $M .(\mathcal{O} \Gamma)$. Then $\mathcal{D}$ contains $\mathcal{O} \Gamma$ as required.

\section{Comments}

(1) The family of integral representations constructed above is by no means exhaustive. For, by construction the representations of Section 1 all lie on the parameter curve $6+u^{2}+v^{2}=u+v+2 u v$. Using the same ideas, other families can be constructed. One can (apparently) always arrange $a$ maps to the "standard element" of order three in the family of the introduction, denote this element by $\tau$. Then a second nonconjugate family of representations is given by

$$
a \mapsto \tau, \quad b \mapsto\left(\begin{array}{ccc}
-5-14 t-16 t^{2}-6 t^{3} & 6+9 t+4 t^{2} & 8+19 t+18 t^{2}+6 t^{3} \\
1-4 t^{2}-6 t^{3} & -1+t+4 t^{2} & -1+t+6 t^{2}+6 t^{3} \\
-2(2+3 t)\left(1+t+t^{2}\right) & 5+5 t+4 t^{2} & 6+13 t+12 t^{2}+6 t^{3}
\end{array}\right) .
$$

In addition, there are apparently sporadic examples, like $u=49$ and $v=21$ yielding the representation

$$
a \mapsto \tau, \quad b \mapsto\left(\begin{array}{ccc}
1 & 20 & 48 \\
0 & -8 & -19 \\
0 & 3 & 7
\end{array}\right)
$$


(2) One can also ask the analogous question for other triangle groups (for allowable $p, q$ and $r$ 's) and the same method can be used. For example, a family of faithful discrete representations of $\Delta(3,4,4)$ (integral for even $t$ ) is given by

$$
a \mapsto\left(\begin{array}{ccc}
1 & 4+3 t^{2} / 4 & 3\left(6-t+t^{2}\right) / 2 \\
0 & -\left(4+t+t^{2}\right) / 2 & -3-t^{2} \\
0 & \left(4+2 t+t^{2}\right) / 4 & \left(2+t+t^{2}\right) / 2
\end{array}\right), \quad b \mapsto\left(\begin{array}{ccc}
0 & 0 & 1 \\
1 & 0 & -1 \\
0 & 1 & 1
\end{array}\right) .
$$

(3) For triangle groups, the representation variety has dimension 2 and the question of integral representations is reduced to a Diophantine analysis applied to output from the application of [4]. However if one passes to subgroups of finite index, one can obtain further integral representations by a bending construction, which we now sketch:

One can pass to a subgroup of index four inside $\Delta(3,4,4)$ to obtain integral representations of $S^{2}(3,3,3,3)$ - a sphere with four cone points all of order 3 . To be specific, fix some even value of $t$, say 4 in the restriction of the representation 2. Denote this by $\rho_{4}$.

On this sphere four cone points, one can find simple closed curves with two cone points on each side, let $\gamma$ be such a curve and suppose (this is often the case) that the characteristic polynomial of $\rho_{4}(\gamma)$ is $\mathbf{Z}$-irreducible. It follows from standard number theory that the centralizer of this matrix in $\operatorname{SL}(3, \mathbf{Z})$ is $\mathbf{Z} \times \mathbf{Z}$ and one can use the standard ideas of bending using this centralizer to give other families of representations of $S^{2}(3,3,3,3)$ which cannot extend up to the original triangle group.

\section{References}

[1] H Bass, Groups of integral representation type, Pacific J. Math. 86 (1980) 15-51 MR586867

[2] B H Bowditch, Atoroidal surface bundles over surfaces, Geom. Funct. Anal. 19 (2009) 943-988 MR2570310

[3] S Choi, W M Goldman, The deformation spaces of convex $\mathbb{R} \mathbb{P}^{2}$-structures on 2orbifolds, Amer. J. Math. 127 (2005) 1019-1102 MR2170138

[4] D Cooper, D D Long, M Thistlethwaite, Computing varieties of representations of hyperbolic 3-manifolds into SL(4, $\mathbb{R})$, Experiment. Math. 15 (2006) 291-305 MR2264468

[5] W M Goldman, Convex real projective structures on compact surfaces, J. Differential Geom. 31 (1990) 791-845 MR1053346

[6] D D Long, A W Reid, Small subgroups of SL(3, Z), to appear in Experiment. Math. 
[7] I Reiner, Maximal orders, London Math. Soc. Monogr. New Ser. 28, The Clarendon Press, Oxford Univ. Press, Oxford (2003) MR1972204 Corrected reprint of the 1975 original, With a foreword by M J Taylor

[8] J-P Serre, Trees, Springer, Berlin (1980) MR607504 Translated from the French by J Stillwell

[9] J Tits, Free subgroups in linear groups, J. Algebra 20 (1972) 250-270 MR0286898

[10] E B Vinberg, V G Kac, Quasi-homogeneous cones, Mat. Zametki 1 (1967) 347-354 MR0208470

[11] S Wang, Representations of surface groups and right-angled Artin groups in higher rank, Algebr. Geom. Topol. 7 (2007) 1099-1117 MR2336251

Department of Mathematics, University of California, Santa Barbara

Santa Barbara CA 93106, USA

Department of Mathematics, University of Texas

Austin TX 78712, USA

Department of Mathematics, University of Tennessee

Knoxville TN 37996, USA

long@math.ucsb.edu, areid@math.utexas.edu, morwen@math.utk.edu

http://math.ucsb.edu/ long, http://www.ma.utexas.edu/users/areid/, http://www. math.utk. edu/ morwen

Proposed: Danny Calegari

Seconded: Benson Farb, Leonid Polterovich
Received: 22 May 2010 Revised: 28 September 2010 